\title{
Supplementary materials for: From Fault Creep to slow and fast Earthquakes in Carbonates
}

\author{
François X. Passelègue ${ }^{1,2}$, Jérôme Aubry ${ }^{3}$, Aurélien Nicolas ${ }^{3}$, Michele Fondriest ${ }^{2}$, Damien \\ Deldicque $^{3}$, Alexandre Schubnel ${ }^{3}$ and Giulio Di Toro ${ }^{2,4}$ \\ ${ }^{1}$ Laboratoire Expérimental de Mécanique des Roches, École Polytechnique Fédérale de \\ Lausanne, Lausanne, Switzerland \\ ${ }^{2}$ SEES, The university of Manchester, Manchester England \\ ${ }^{3}$ Laboratoire de Géologie de l'École Normale Supérieure, Paris, France \\ ${ }^{4}$ Dipartimento di Geoscienze, Università degli Studi di Padova, Padua, Italy
}

\section{Sample used in this study}

Cylindrical samples (diameter: $40 \mathrm{~mm}$, length: $90 \mathrm{~mm}$ ) were cored from dolostone blocks of the Mendola Formation (Upper Triassic in age), a dolomitized platform carbonate with crystal size in the range 20 to $300 \mu \mathrm{m}$ and larger crystals filling diagenetic pores (Fondriest et al., 2017). These dolostones are equivalent to the Upper Triassic-Jurassic dolomitized carbonates composing the seismogenic layer of the Central Apennines and were characterized in terms of acoustic and elastic properties in previous studies (Fondriest et al., 2017). The rock cylinders were saw-cut to create an experimental fault at an angle of $30^{\circ}$ with respect to $\sigma_{1}$ (principal stresses are denoted $\sigma_{1}>\sigma_{2}=\sigma_{3}$ ). Fault surfaces were roughened first with grinder and then with coarse sandpaper (grit number P240, which corresponds to $\pm 50 \mu \mathrm{m}$ in surface roughness) using ethanol to avoid frictional heating during sample preparation. All experiments were conducted on fault surface presenting the same initial geometry and roughness (Figures S1a and S1b).

\section{Materiel and methods}

\section{Experimental apparatus}

The apparatus used in this study is a tri-axial oil medium loading cell $\left(\sigma_{1}>\sigma_{2}=\sigma_{3}\right)$ built by Sanchez Technologies. The confining pressure is directly applied by a volumetric servo-pump up to a maximum of $100 \mathrm{MPa}$. The axial stress is controlled independently by an axial piston controlled by a similar servo-pump. The axial stress can reach $680 \mathrm{MPa}$ on $40 \mathrm{~mm}$ diameter samples. Both confining and axial pressure are controlled and measured with a resolution of $0.01 \mathrm{MPa}$. Axial shortening is measured by averaging the values recorded on three capacitive gap sensors located externally. These sensors record both the sample deformation and that of the apparatus. The resolution of these measurements is 0.1 micron. Both pressure and displacement data are 
recorded at sampling rates ranging between 10 -to-1000 $\mathrm{Hz}$ during experiments. More details can be found in Brantut et al., (2011). Note that because of our sample geometry, increasing the differential stress leads to an increase of both shear and normal stresses.

Acoustic monitoring system

During experiments, acoustic activity was monitored through 15 piezo-ceramic sensors which consist of a PZT crystal (PI ceramic PI255 $0.5 \mathrm{~mm}$ thick and of diameter $5 \mathrm{~mm}$ ) contained in a brass casing (Figure S1b). The sensors were glued directly on the samples with cyanoacrylate adhesive. Acoustic waveforms were recorded with two different techniques (Passelegue et al., 2017). First, each unamplified signal was relayed to a digital oscilloscope allowing for the recording of macroscopic stick-slip events within a time window of $6.5 \mathrm{~ms}$ at $10 \mathrm{MHz}$ (Passelegue et al., 2017). Second, to record low amplitude acoustic emissions activity, signals were amplified at $45 \mathrm{~dB}$ though pre-amplifiers. Amplified signals were then relayed to a trigger logic box. Using this second system, AEs were recorded if at least 4 sensors recorded an amplitude larger than a given threshold, that is set at 0.001 Volts. The complete waveform catalogue was then manually analyzed to remove possible triggers from background noise.

\section{Strain gages measurements}

In addition to the record of regular mechanical data, four strain gauges $1.5 \mathrm{~cm}$ equally spaced and recording preferentially shear strain $\varepsilon_{\tau}$ were glued at $4 \mathrm{~mm}$ from the fault plane along the fault strike (Figure S1b). Mechanical data and strain measurements were recorded at $2.4 \mathrm{kHz}$ sampling rate. In addition to classical strain gages, a Wheatstone bridge strain gages was glued directly on the rock sample close to the fault plane (Figure S1b). The Wheatstone bridge is composed of four resistors ( $\Omega=350$ ohms) measuring together the differential strain $\left(\varepsilon_{1}-\varepsilon_{3}\right)$. The signals are relayed to a high frequency strain gage amplifier allowing up to $10 \mathrm{MHz}$ sampling rate. The strain gages are calibrated using low frequency stress and strain measurements during the elastic part prior to each STE, assuming a constant Young's modulus ( $E=64 \mathrm{GPa}$ ) for Dolomite. Assuming linear elasticity, the measurement at high sampling rate of the differential strain is converted into the dynamic evolution of the differential stress (i.e., $\sigma_{1}-\sigma_{3}$ ) during STE. The assumption of linear elasticity during short time intervals remains robust because damage is localized during stick-slip instability in comparison with intact specimens and do not affect the bulk of the granite at the measurement location.

\section{Rupture properties}

\section{Slip measurement}

Axial displacements $\left(D_{\mathrm{ax}}\right)$ were recorded through three gap sensors located outside the cell with a resolution of $0.1 \mu \mathrm{m}$ at $2.4 \mathrm{k}$ samples per second. External data (collected using the gap sensors) are corrected using the axial deformation of the sample measured using strain gages glued 
directly on the rock sample. Up to four pairs of strain gages can be used during each experiment. Each pair of strain gages is composed of two resistors ( $\Omega=120$ ohms) measuring respectively the axial and the radial strain, corresponding to $\varepsilon_{1}$ and $\varepsilon_{3}$ in the selected frame of reference. Strains are recorded continuously at a sampling rate of $2.4 \mathrm{kHz}$. Using these measurements, we can estimate the elastic constants of the rock during the elastic part of the experiments and correct the shortening measured externally from the rigidity of the apparatus using the relation:

$$
\varepsilon_{a x}^{F S}=\varepsilon_{a x}^{\text {sample }}+\frac{\Delta \sigma}{E_{a p}} \quad \text { Equation 1 }
$$

where $\varepsilon_{a x}{ }^{F S}$ is the average axial strain measured on gap sensors, $\varepsilon_{a x}^{\text {sample }}$ is the axial strain of the sample, $\Delta \sigma$ is the differential stress applied during the loading stage and $E_{a p}$ is the rigidity of the apparatus. The rigidity of the apparatus was estimated for each conditions tested, and depends of the applied confining pressure and of the ambient temperature.

Using the measurement of the axial shortening by capacitive gap sensors located externally combined with axial strain gage measurements, we are able to estimate the axial displacement from

$$
D_{a x}=\varepsilon_{a x}^{\text {sample }} L=\left(\varepsilon_{a x}^{F S}-\frac{\Delta \sigma}{E_{a p}}\right) L \quad \text { Equation 2 }
$$

where $L$ is the length of the rock sample. The finite displacement along the fault during stick-slip instabilities, called also final displacement, is then estimate using a simple projection assuming

$D_{f}=D_{a x} / \cos \theta$

Equation 3

All displacements discussed in the main text correspond to displacement along the fault $\left(D_{f}\right)$.

\section{Estimation of the fault's elastic stiffness}

For each events, the fault elastic stiffness was computed from axial strain measurement using strain gages. Fault elastic stiffness was measured following

$K_{f}=\frac{\Delta \sigma}{L_{f} \varepsilon_{1}}$

Equation 4

where $L_{f}$ is the length of the fault. Assuming these estimations, our experimental results show that the fault system elastic stiffness increases with the confining pressure, but also only with the ambient temperature (Figure S2a). Note that while this fault elastic stiffness is expected to control the rupture mode when its becomes greater than the stiffness of the apparatus (Leeman et al., 2016) (Figure S2b), it is not the case in our experiments and large $K_{f}$ recorded at $65^{\circ} \mathrm{C}$ temperature and 30 and $60 \mathrm{MPa}$ confining pressure induces only slow rupture while $K_{f}>K_{a p}$ (Figure $\mathrm{S} 2$ ) 


\section{Estimation of the slip velocities}

Slip velocities were computed following two different methods due to technical limitations. For stable slip and slow ruptures, slip velocities were estimated from the displacement measurement corrected from the elastic stiffness of the apparatus (Equation 3 ), and consist simply in the derivative of $D_{f}$ with time.

During dynamic rupture, our sampling rate of the slip measurement does not allow a direct estimation of the slip velocity because most of the slip occurs within 20 microseconds. In these conditions, an estimate of the slip velocity is computed from the static slip measured at $2.4 \mathrm{kHz}$, divided by the weakening time recorded by dynamic strain gages (Passelegue et al., 2016; Brantut et al., 2016). This estimate of the slip velocity has been confirmed recently using a laser vibrometer which now allow slip velocity measurement at high sampling rate. However, this system was not available at the stage of the experiments presented here.

\section{Inversion of the dynamic rupture speed}

While the rupture speed during slow rupture front was simply compute as a function of the travel time of the rupture through the array of strain gages located along the fault (Figures S1b and 2a), the inversion of the rupture speed during fast rupture required an inversion from the acoustic data. Due to the 3D experimental geometry, a complex mixed-mode rupture can develop. Under the applied loading conditions, the principal slip direction is parallel to the fault length (main axis of the ellipsoidal fault). As a consequence, a mode II (in-plane) rupture will propagate along the fault length, while a mode III (antiplane) rupture will propagate along its width. This implies that the rupture velocity, $V_{r}$, along the fault length (mode II) is such that $V_{r}<C_{R}$ during a sub-Rayleigh rupture and $C_{S}<V_{r}<C_{P}$ during a supershear rupture $\left(C_{R}, C_{S}\right.$ and $C_{P}$ being the Rayleigh, shear and compressional elastic wave velocities respectively). In the Mode-III direction, along the fault width, rupture velocity is always $V_{r}<C_{R}$. For simplicity we approximate the shape of the rupture front as circular at sub-Rayleigh velocity and as elliptical at supershear velocity, where the ratio of the two axes corresponds to the ratio of the velocities in the inplane direction.

For each STE, the first wave arrival recorded on each sensor was manually picked for better accuracy. The location and the rupture velocity are then inverted using a least-squares method comparing the experimental arrival times to theoretical arrival times calculated using the approximate rupture front geometry described above. To be more precise, the theoretical arrival times are calculated as a function of (i) the possible rupture velocity $V_{r}$ along strike (ii) the rupture front geometry (circular rupture front up to $V_{r}=C_{R}$, elliptical above $C_{S}$ ), (iii) the time of initiation of the event, and finally (iv) the positions of the sensors relative to the nucleation zone of rupture. We assume that the lowest residual time outputs the best solution for (i) the location of the nucleation zone, (ii) the time of initiation and (iii) the average rupture velocity along strike. Thereafter the value of the rupture velocity at a given point of the fault can be estimated during supershear event using 


$$
V_{r(X, Y)}=\frac{1}{\sqrt{\frac{\cos ^{2} \alpha}{C_{S}^{2}}+\frac{\sin ^{2} \alpha}{V_{I I}^{2}}}} \quad \text { Equation } 5
$$

where $V_{\|}$is the rupture velocity parallel to the slip direction and $\alpha$ is the angle between the coordinates of the given point $(X, Y)$ and the mode-III direction perpendicular to the slip direction (Passelègue et al., 2016).

\section{Complementary experiments and microstructures}

Recent experimental studies have shown than in calcite, the transition from stable to unstable slip is explained by the activation of plastic processes. To understand further the link between the microstructures and the mode of slip, the formation of nanograins, and the mechanisms of deformation involved during the interseismic stage of the experiments, we conducted several specific experiments. The first experiments consisted in a simple elastic loading of the experimental fault, submitted at the three confining pressure and each temperature tested $\left(30,65\right.$ and $\left.100{ }^{\circ} \mathrm{C}\right)$. The fault was loaded up to $90 \%$ of the expected differential stress leading to instability. Then, this stress was keep constant during a period ranging from 10 minutes to 1 hour. The fault was then unloaded and the sample removed from the apparatus.

The experimental results of these experiments highlighted that fault slip during constant load was observed only at low confining pressure and low temperature conditions, where the elastic fault stiffness present the lowest values. At $100{ }^{\circ} \mathrm{C}$, conditions were dynamic rupture were observed in the experiments presented in the main text, the fault remains completely stick during the entire constant load experiments (no axial shortening), which suggest a pure elastic behavior.

Secondly, the post mortem microstructures conducted after these experiments highlighted that fault creep induced at low confining pressure induces the formation of mirror-like surface and gouge production along clivage. At $100{ }^{\circ} \mathrm{C}$, where no slip was recorded, fault surface looks identical to the starting material and only very rare nanograins aggregates were observed (Figure S3). These results suggest that the nanograins observed after both slow rupture and dynamic ruptures are not present before the onset of the first instability at $100{ }^{\circ} \mathrm{C}$ and that plastic processes are not dominant along fault during the loading part of the experiments conducted at $100^{\circ} \mathrm{C}$, at the opposite of the behavior observed in calcite.

\section{Post rupture experiments microstructures}

\section{Post mortem fault surfaces}

Remarkably, the fault surfaces recovered from experiments conducted at different ambient temperature and which have experienced stable slip and slow rupture or dynamic rupture are very different even at the naked eyes (Figures S4a, S4b and S4c). Fault surfaces recovered from experiments where stable slip and 
slow ruptures occurred have highly light reflective patches visible (Figure S4b). Instead, these extended mirror-like structures are not found on fault surfaces which experienced fast ruptures (Figure S4c). In fact, these fault surfaces are on average rougher but still characterized by the presence of limited in extension and discontinuous smooth patches with visible slicken-lines (See Figure S4d-I for SEM images at the same scale). This remarkable microstructural difference implies that weakening during faulting was induced by distinct processes in slow versus fast rupture propagation. Ponctual chemical analysis by energy-dispersive spectroscopy (EDS) shows that residual dolomite grains after dynamic rupture events present similar fraction of $\mathrm{O}$ and $\mathrm{Ca}$ or $\mathrm{O}$ and $\mathrm{Mg}$, with residual fraction of bot $\mathrm{Mg}$ and Ca similarly to analysis conducted after high velocity friction experiments (Green et al., 2014). These punctual analyses could suggest that the residual grains are formed of oxydes ( $\mathrm{MgO}$ and $\mathrm{CaO}$ ) due to fast rupture events, which may suggest decarbonation-assisted melting (Brooker et al., 1998) of the slip surface due to frictional heating. However, it remains not clear if melting occurs before decarbonation or after.

\section{Determination of weakening velocity for flash heating}

As stated in the caption of the figure $4 d$, the weakening velocity allowing flash heating depends of the hardness of dolomite, of the weakening temperature (decarbonation or melting temperature for exemple) and of the size of the asperities. While we have an idea of both the hardness and the weakening temperature of dolomite, an estimate of the size of the asperities acting along the fault prior instabilities remains hard to estimate. From literature, this size depends of minerals, and of the normal stress acting along the fault. In the manuscript, we consider a size of asperities of 50 microns based on microstructural analysis and roughness measurement of the post mortem fault surfaces which experienced stick slip events and strong weakening (Figure S5). However, this calculation is made in the text to show that the slip rate estimated during dynamic rupture is largely faster than critical values allowing flash temperature (Goldsby and Tullis, 2011; Tisato et al., 2012; Violay et al., 2014; Passelegue et al., 2014). According to our estimates of slip rates reached during dynamic ruptures, we can estimate the minimum size of asperities which allow decarbonation of dolomite following

$\begin{array}{ll}d_{\min }=\frac{(\rho C)^{2}\left(T_{w}-T_{0}\right)^{2} \pi \alpha}{V_{S}} & \text { Equation } 6\end{array}$

with $V_{s}$ the average rupture speed of all dynamic rupture events, $T_{w}$ the weakening temperature corresponding to decarbonation. Doing this calculation, we can state that asperities larger than 0.16 microns are expected to decarbonate during fast ruptures, which is largely below the range of the imposed and measured roughness, and below the size of the asperities imaged by SEM analysis along post-mortem fault surfaces. 


\section{Supplementary figures}

a.

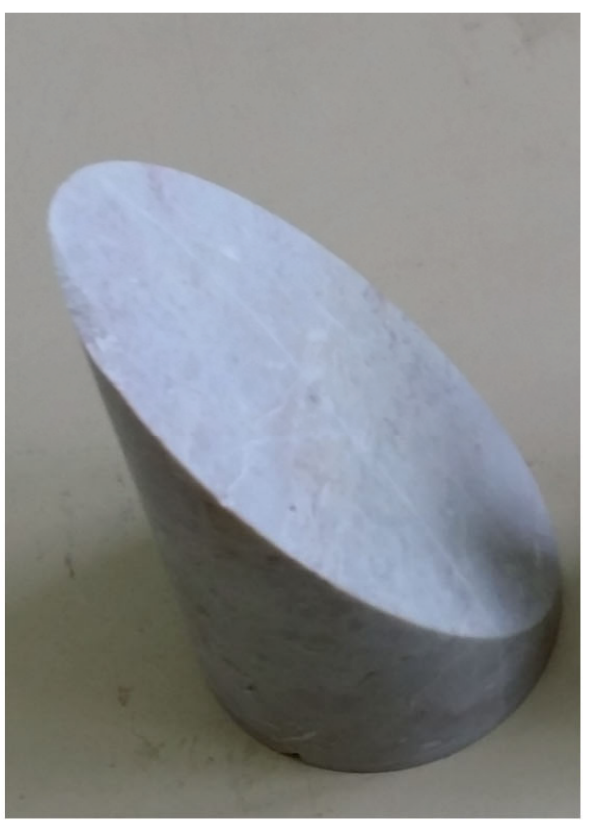

b.

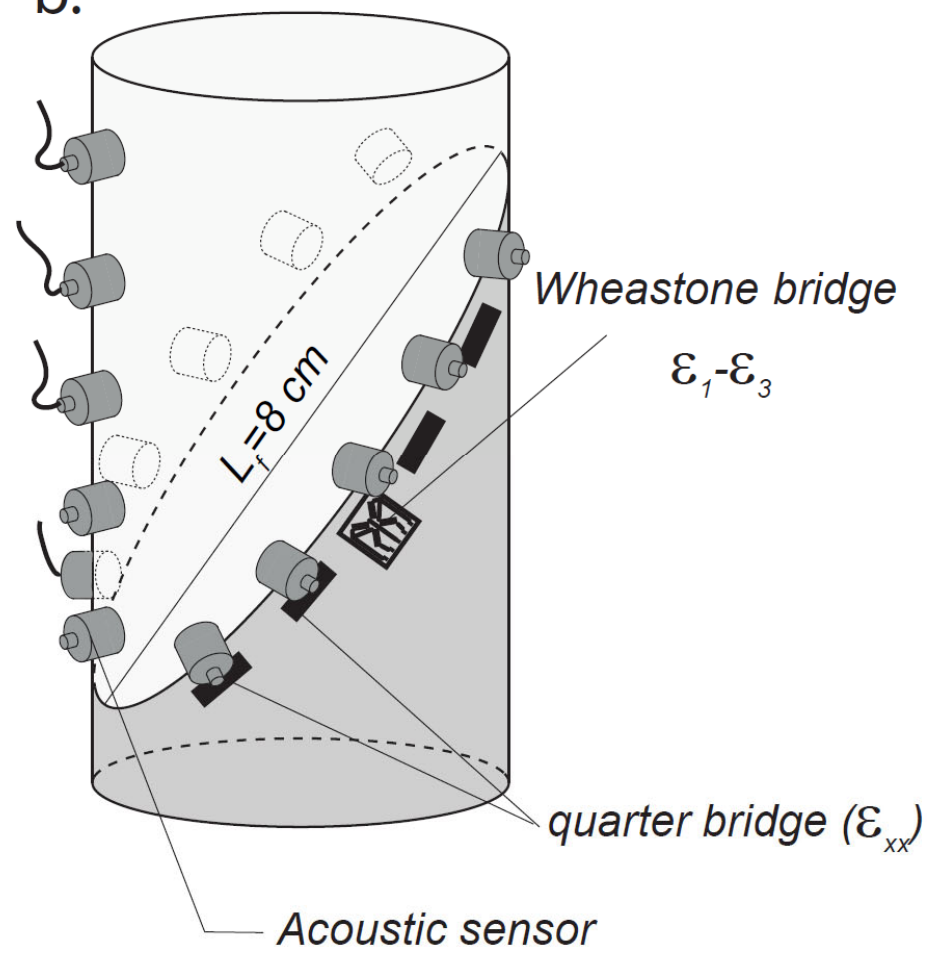

Figure 1. Starting material and experimental setup used in this study. (a) Picture of a saw cut specimens of natural dolomite. (b) Scheme presenting the fault geometry and the sensors used to invsetigate rupture processes. Quarter bridge were reccorded at $2.4 \mathrm{kHz}$ sampling rate, allowing to track slow rupture front. Both Wheastone bridge and acoustic signals were reccorded at $10 \mathrm{MHz}$ sampling rate, allowing to track fast motions during dynamic instabilities. 

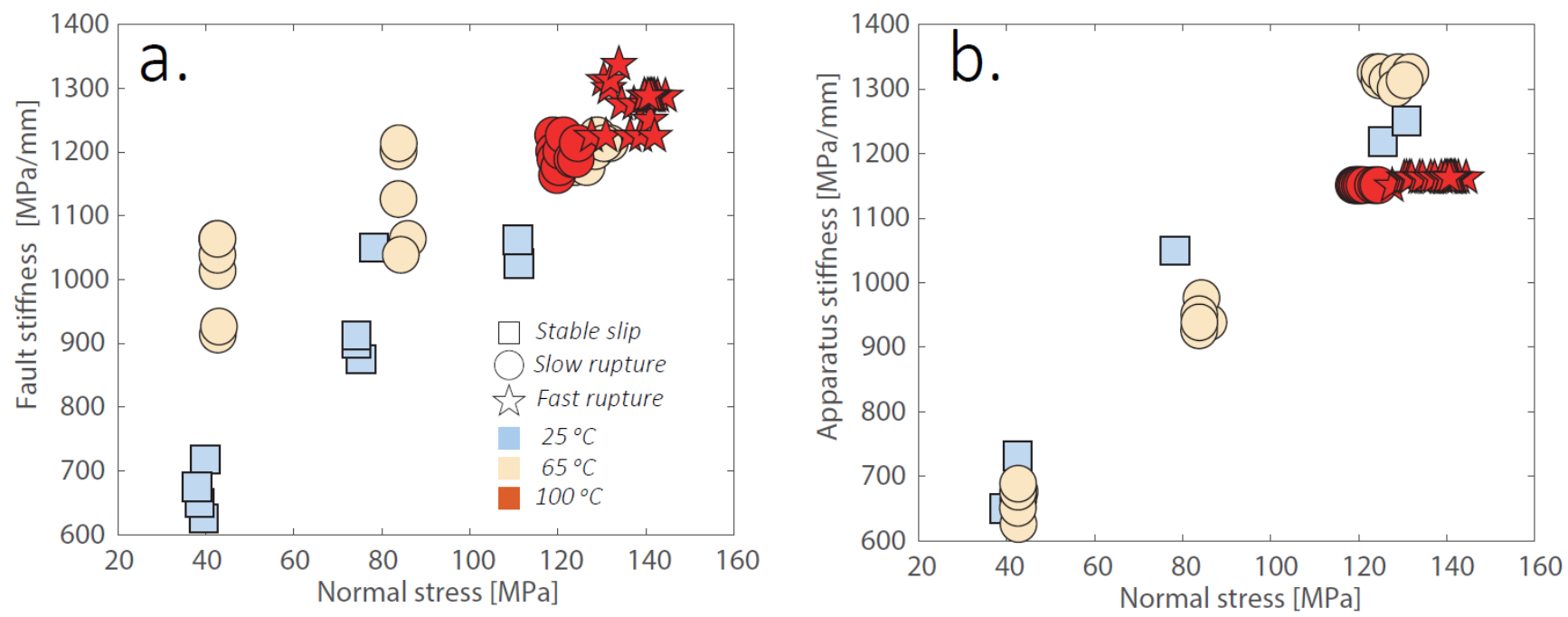

Figure 2. Relation between the state of stress, the bulk temperature and the elastic loading stiffness of the fault (a) and the stiffness of the apparatus (b). Note that the ratio of the two stiffness measured is expected to be a proxy for the mode of slip. 


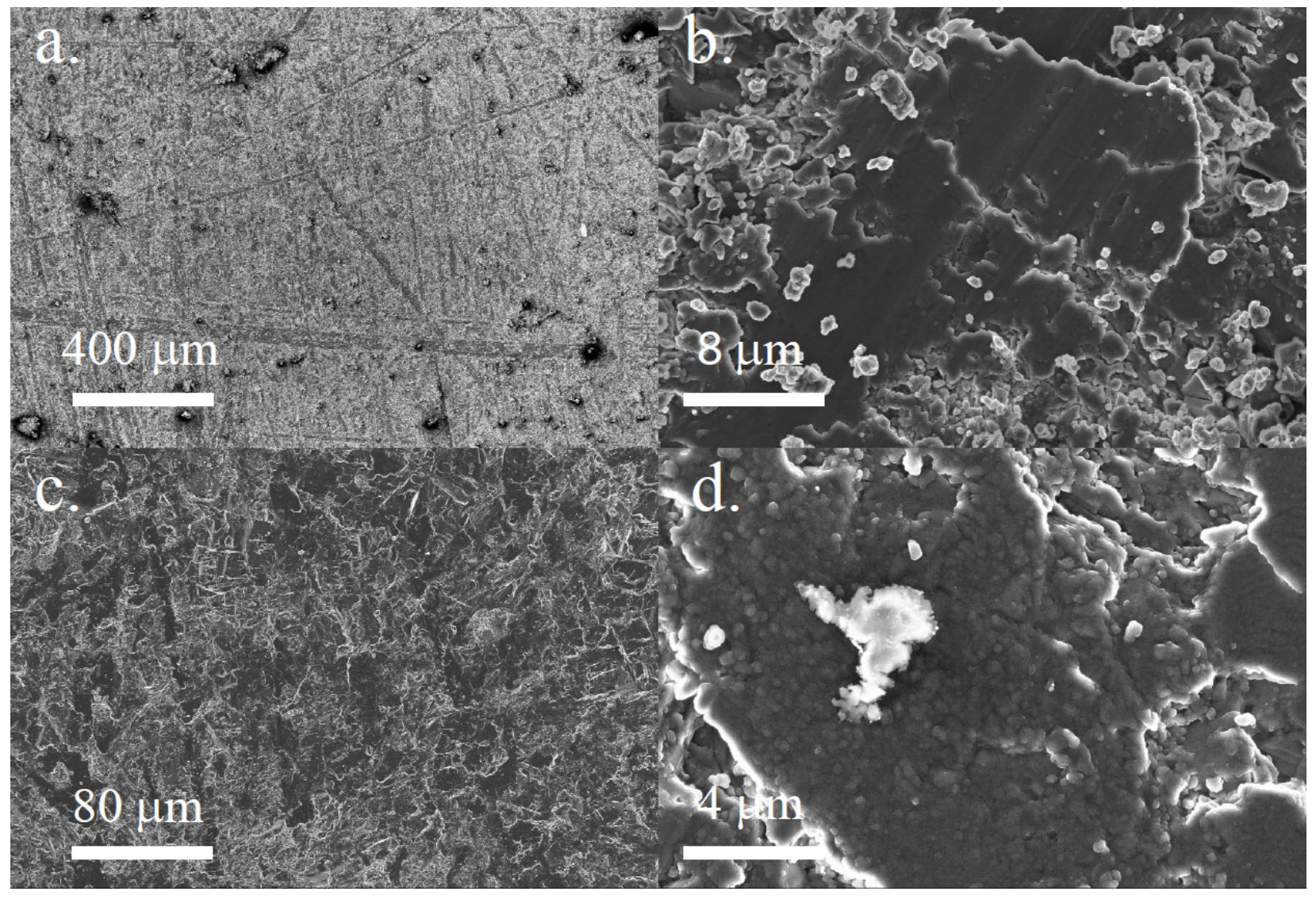

Figure 3. Post mortem microstructures observed after elastic loading of the fault at 90 percent of the peak strength at $60 \mathrm{MPa}$ confining pressure and room temperature (a. and b.), and at $60 \mathrm{MPa}$ and $100{ }^{\circ} \mathrm{C}$ (c and d). Note that $d$ corresponds to the only evidence of "nanograins" observed on the fault surface after the elastic loading, suggesting that nanograins are absent, and not required, prior the first dynamic instability. 


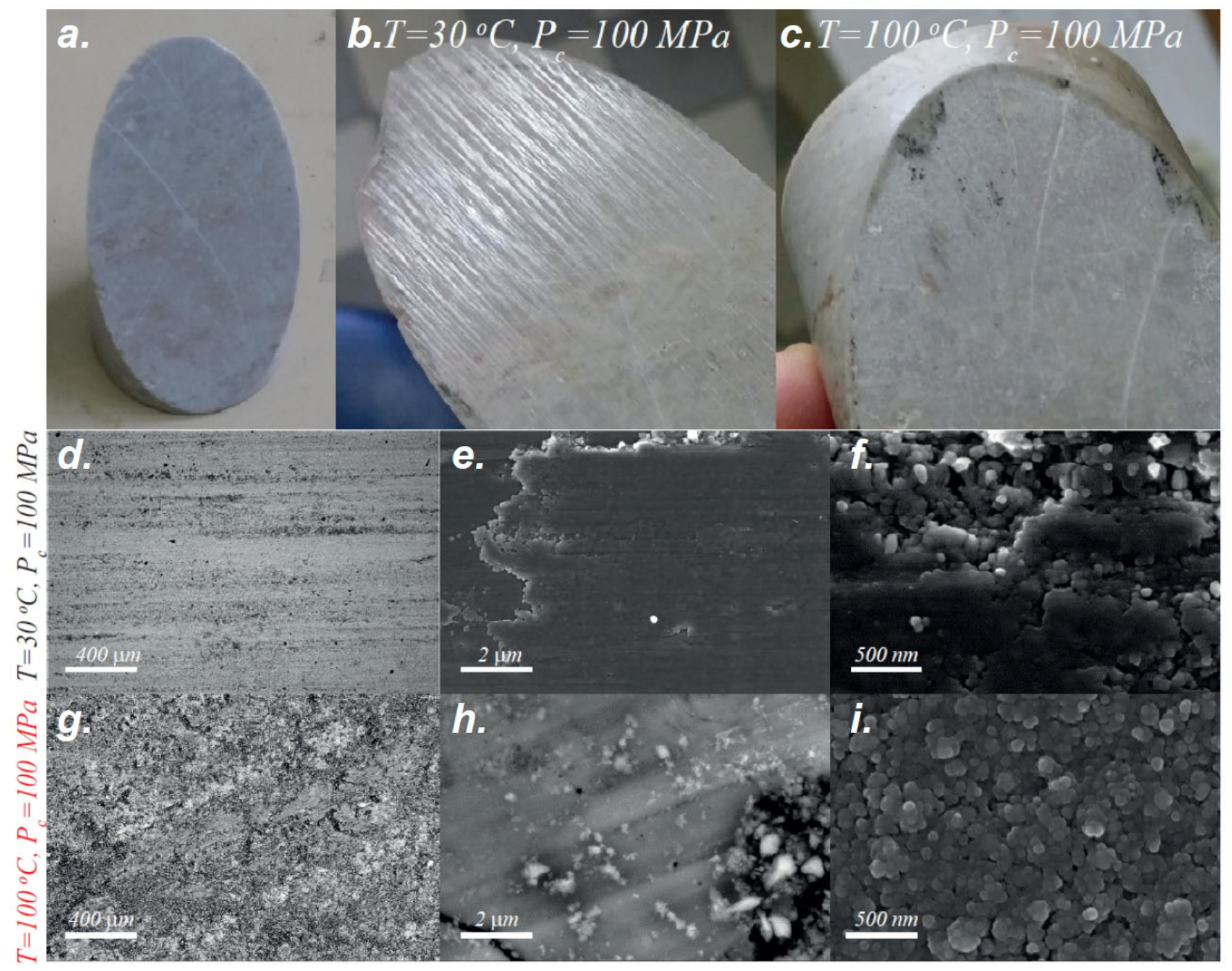

Figure 4. Post mortem fault microstructures. (a), (b) and (c) corresponds to picture of the fault before, after stable slip or slow rupture, and after dynamic rupture, respectively. Note that macroscopic mirror- 
like surface are observed only after stable slip and slow rupture events. (d), (e) and (f) correspond to micrographs of the fault surfaces after stable slip events induced at $100 \mathrm{MPa}$ confining pressure and at room temperature. (g), (eh) and (i) correspond to micrographs of the fault surfaces after dynamic events.

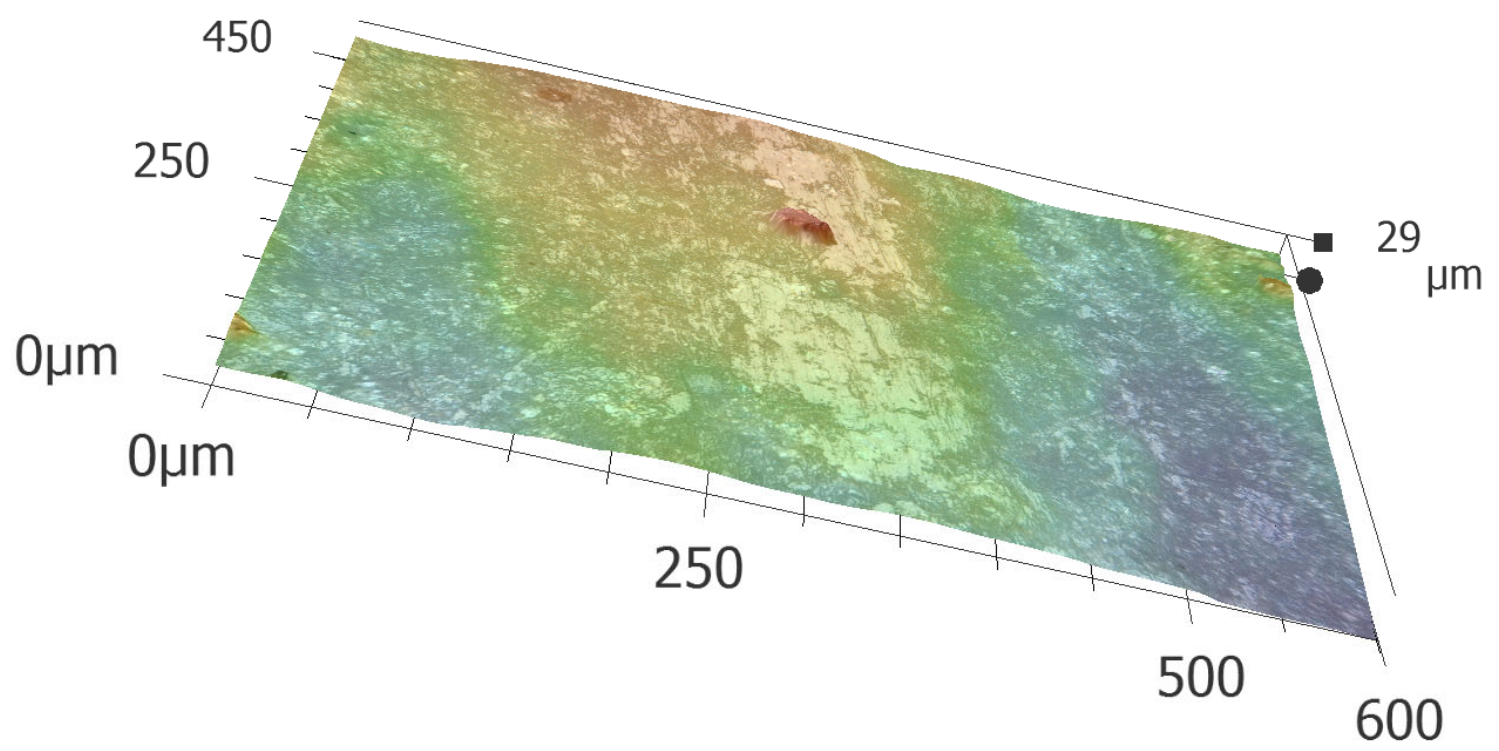


Figure 5. Roughness of the fault that has undergone dynamic rupture events. Note that contact sliding area can be estimate around 25 to 100 microns in size. 